\title{
Temporally and spectrally resolved imaging of laser-induced plasmas
}

\author{
J. Siegel, G. Epurescu, A. Perea, F. J. Gordillo-Vázquez, J. Gonzalo, and C. N. Afonso \\ Laser Processing Group, Instituto de Óptica, Consejo Superior de Investigaciones Cientificas, Serrano 121, 28006 Madrid, Spain
}

Received June 18, 2004

\begin{abstract}
We report a hybrid imaging technique capable of performing measurements of the spatial, temporal, and spectral emission characteristics of laser-induced plasmas by use of a single detection system. We apply this technique to study the plasma produced by laser ablation of $\mathrm{LiNbO}_{3}$ and observe phenomena not seen in such detail with standard instruments. These include extreme line broadening up to a few nanometers accompanied by self-absorption near the target surface, and expansion dynamics that differ strongly between the different species. Overall, the wealth of quantitative information provided by this novel technique sheds new light on processes occurring during plasma expansion. () 2004 Optical Society of America

OCIS codes: $110.0110,160.3730,260.6580,300.3700,300.6500$.
\end{abstract}

Plasma emission spectroscopy (PES) is a powerful optical tool for remote material analysis and is receiving growing interest in a wide range of research areas. $^{1}$ It consists of irradiating a material with a high-energy laser pulse and measuring the emission spectrum of the laser-induced plasma formed near the material surface. Because each atom in every state of excitation has its own characteristic spectral fingerprint, PES (also known as laser-induced breakdown spectroscopy) potentially permits the identification of the elemental composition of a material. PES can also be applied to the study of plasmas produced during pulsed laser deposition, because the physical properties of the deposited films strongly depend on the plasma expansion dynamics. ${ }^{2}$ In its simplest form, PES is performed with a spectrometer, and time resolution can be added by use of a suitable photomultiplier. A drawback of this approach is the restriction to single-point measurements, providing the spectral information of only a single spatial point of the plasma or a space-averaged emission spectrum of the entire plasma. However, space resolution is clearly desirable since the plasma expands from the sample surface and the intensities of the emission lines become a function of space and time. When using an imaging detector, for instance, a time-gated intensified charge-coupled device (ICCD), snapshots of the expanding plasma at different stages of the expansion process can be obtained, but spectral information is lost. ${ }^{3}$ Spectral information can be added by use of another imaging approach based on Fourier-transform visible spectroscopy, providing the emission spectrum for each pixel of the image. ${ }^{4}$ However, this technique is time consuming because it relies on a mechanically driven interferometer and requires an image to be collected for each interferometer step (100 images in their case).

In this work we combine the ability of PES to distinguish between different species with the capability of time-gated imaging to localize the species in space and time. This is, to the best of our knowledge, the first time that precise measurement of all three parameters (spectral, spatial, and temporal) of the emission characteristics of a laser-induced plasma and their interdependence are incorporated into a single imaging detector. This detector, which can acquire the complete spatial evolution of the spectral plasma emission lines induced by a single laser pulse, is based on an imaging spectrograph and a time-gated ICCD. Unlike the elegant fiber-based approach of Pérez-Tijerina et al., ${ }^{5}$ which is able to reconstruct a low-spatial-resolution twodimensional (2D) image (25 pixels) of the plasma with wavelength resolution, we deliberately trade in lateral resolution of the plasma for high resolution in the expansion direction, limited only by the ICCD pixel size $(24 \mu \mathrm{m})$. Harilal et al. ${ }^{6}$ reported a system similar to ours, but theirs requires the image to be scanned over the entrance aperture. It therefore lacks the benefits of parallel acquisition of emission spectra along the entire expansion direction.

The experimental apparatus is shown in Fig. 1. An $\operatorname{ArF}$ excimer laser $(\lambda=193 \mathrm{~nm}$, pulse duration of $20 \mathrm{~ns}$ ) is focused on a target located inside a vacuum chamber $\left(10^{-6}\right.$ mbars $)$ generating a plasma that expands along the normal to the target surface ( $z$ direction). The emitting plasma is imaged onto a Czerny-Turner imaging spectrograph $(f / 4$ aperture ratio, $25-\mathrm{cm}$ focal length, $0.15-\mathrm{nm}$ resolution, 2.5-nm/mm dispersion) by means of two quartz lenses $\left(f_{1}=160 \mathrm{~mm}, f_{2}=100 \mathrm{~mm}\right)$ and a periscope. The purpose of the periscope is to rotate the plasma image by $90^{\circ}$ such that the expansion direction $(z)$ lies along the orientation of the entrance slit of the spectrograph. For the results presented here, a 475-nm long-pass filter was installed in front of the spectrograph to prevent second-order diffraction of short-wavelength light. The spectrograph incorporates three gratings (G1, 600 grooves $/ \mathrm{mm}$, blaze of $750 \mathrm{~nm} ; \mathrm{G} 2$, 300 grooves/mm, blaze of $1700 \mathrm{~nm}$; G3, 150 grooves/ $\mathrm{mm}$, blaze of $500 \mathrm{~nm}$ ). At its output the spectrograph produces a one-dimensional (1D) spatial and spectral image of the expanding plasma, in which the vertical axis corresponds to expansion direction $z$ and the horizontal axis to wavelength $\lambda$ of the emission of the species. In addition, if the gratings 


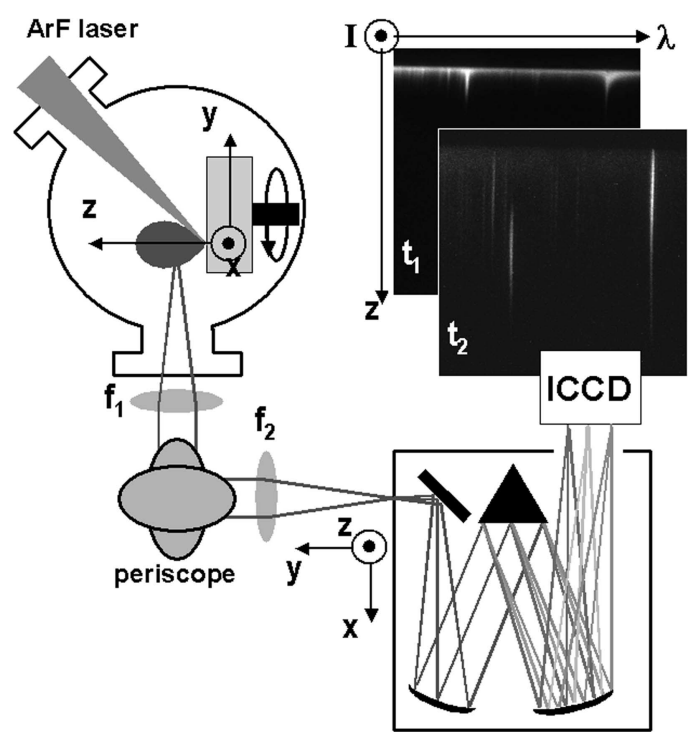

Fig. 1. Experimental setup for time-resolved plasma imaging with spectral resolution of laser-induced plasmas.

are operated in reflection rather than in diffraction and the entrance slit is fully open, a $2 \mathrm{D}$ spatial image of the plasma can be formed. At the output of the spectrograph, in the image plane, a time-gated ICCD camera is installed. This ICCD has a spectral range from 180 to $870 \mathrm{~nm}$, a resolution of $512 \times 512$ pixels, with an effective pixel size of $24 \mu \mathrm{m}$, an analog-todigital resolution of $16 \mathrm{bits}$, and a minimum gate width of $2 \mathrm{~ns}$. A delay generator is employed to delay the time gate of the ICCD with respect to the laser pulse by a user-defined value.

To demonstrate the flexibility, sensitivity, and large spectral window of our system, we acquired four time-gated images of the expanding plasma of $\mathrm{LiNbO}_{3}$, each obtained by irradiation with a single laser pulse (fluence of $1.2 \mathrm{~J} / \mathrm{cm}^{2}$ ). Figures 2(a) and 2(b) show two images obtained in the $2 \mathrm{D}$ spatial imaging mode and Figs. 2(c) and 2(d) show two images obtained in the $1 \mathrm{D}$ spatial and spectral imaging mode. The images were obtained with a constant gate width $(\Delta t=30 \mathrm{~ns})$ but different delay times $\left[t_{1}=50 \mathrm{~ns}\right.$ in Figs. 2(a) and 2(c) and $t_{2}=140 \mathrm{~ns}$ in Figs. 2(b) and 2(d)] with respect to the laser pulse. The different stages of the plasma expansion process from the target surface $(z=0 \mathrm{~mm})$ can be appreciated in the 2D spatial images [Figs. 2(a) and 2(b)], showing a directional expansion into the $z$ direction with a relatively low angular spread. The apparent blur at the target surface is caused by chromatic aberrations occurring in the two lenses used, due to the broad spectrum imaged (475-850 $\mathrm{nm}$ ).

By switching to the $1 \mathrm{D}$ spatial and spectral imaging mode, operating grating G3 in diffraction, and using an entrance slit width of $50 \mu \mathrm{m}$, we obtained the corresponding images shown in Figs. 2(c) and 2(d). The vertical axis still corresponds to the $z$ axis of plasma expansion, but the horizontal scale now represents the wavelength of the emitted light. The brightness of each image pixel corresponds to the local emission intensity at the given delay and constitutes the third image dimension. The slight tilt of the near-surface emission with respect to the horizontal plane is caused by chromatic aberration. In the early state of expansion [ $t_{1}$, Fig. 2(c)] a continuous spectrum shows up near the target surface, which can be attributed to a bremsstrahlung generated by deceleration of plasma electrons. $^{7}$ In addition, three individual emission lines can be clearly identified in Fig. 2(c). The lines at $610.3 \mathrm{~nm}\left(3 d^{2} D_{3 / 2,5 / 2} \rightarrow 2 p^{2} P_{1 / 2,3 / 2}\right)$ and $670.7 \mathrm{~nm}$ $\left(2 p^{2} P_{3 / 2,5 / 2} \rightarrow 2 s^{2} S_{1 / 2,3 / 2}\right)$ correspond to radiative transitions of excited $\mathrm{Li}$ neutrals $\left(\mathrm{Li}^{*}\right)$. The 777-nm line $\left(3 p^{5} P_{3,2,1} \rightarrow 3 s{ }^{5} S_{2}\right)$ corresponds to transitions of excited oxygen neutrals $\left(\mathrm{O}^{*}\right)$. Besides, a multitude of emission lines due to $\mathrm{Nb}$ neutrals $\left(\mathrm{Nb}^{*}\right)$ are present, but these are barely perceivable because they spatially overlap with the bremsstrahlung.

At a later stage of the expansion process $\left[t_{2}\right.$, Fig. 2(d)] the emitting species expand further and individual $\mathrm{Nb}^{*}$ lines can be resolved. The fact that the intensity of $\mathrm{Nb}^{*}$ lines is much lower than that of $\mathrm{Li}^{*}$ lines, together with a decreasing sensitivity of typical photocathodes in the infrared region where $\mathrm{O}^{*}$ emit, makes the simultaneous detection of all species involved a challenge. However, as can be seen in Fig. 2, our system is capable of recording $\mathrm{Li}^{*}, \mathrm{O}^{*}$, and $\mathrm{Nb}^{*}$ lines simultaneously due to the large dynamic range of our detector and allows two entirely different expansion dynamics to be discerned. It can be seen that $\mathrm{O}^{*}$ is ejected from the target at only the early stages of the ablation process since the distribution of the $\mathrm{O}^{*}$ line $90 \mathrm{~ns}$ after the laser pulse [ $t_{2}$ in Fig. 2(d)] shows a distinct gap to the target surface. In contrast, $\mathrm{Li}^{*}$ and $\mathrm{Nb}^{*}$ are still emitted from the target surface at this delay.

Spectral resolution can be improved by use of a grating with more grooves per mm. Figure 3(a) shows an image obtained with a single laser pulse with G1. The time gate was extended to $5 \mu \mathrm{s}$ to integrate over all stages of expansion. The multitude of $\mathrm{Nb}^{*}$ lines particularly in the 570-600-nm spectral range can be clearly seen. The strong $\mathrm{Li}^{*}$ line at $610.3 \mathrm{~nm}$ extends
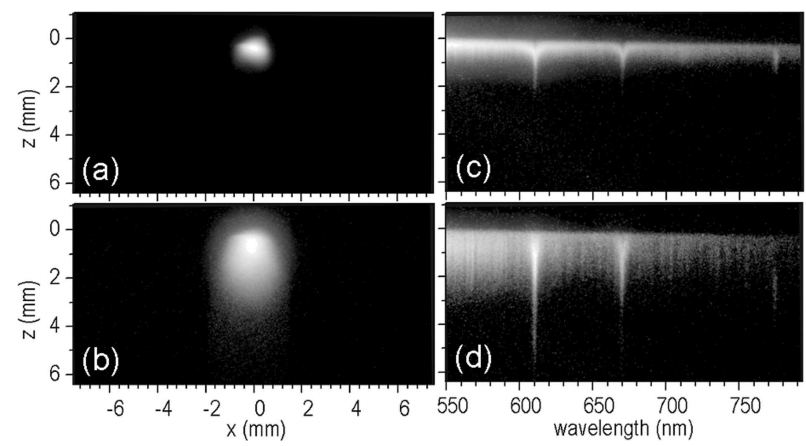

Fig. 2. Time-gated images of the plasma expanding from the surface of a $\mathrm{LiNbO}_{3} \operatorname{target}(z=0 \mathrm{~mm})$, each obtained by irradiation with a single laser pulse by use of (a), (b) the 2D spatial imaging mode and the (c), (d) 1D spatial and spectral imaging mode. The gate width was constant $(\Delta t=$ $30 \mathrm{~ns}$ ), and the gate delay with respect to the laser pulse was changed from (a), (c) $t_{1}=50 \mathrm{~ns}$ to (b), (d) $t_{2}=140 \mathrm{~ns}$. The images are represented in a logarithmic intensity scale to compensate for the large differences in emission intensities between the different species $\left(\mathrm{Li}^{*}, \mathrm{O}^{*}\right.$, and $\left.\mathrm{Nb}^{*}\right)$. 


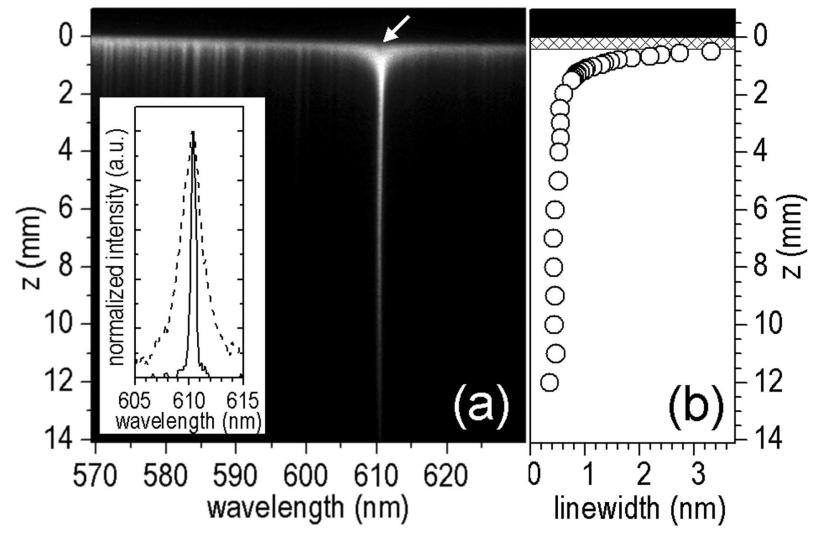

Fig. 3. (a) Time-integrated 1D spatial and spectral high-resolution image of the expanding plasma obtained by irradiation with a single laser pulse. The inset shows intensity profiles of the $\mathrm{Li}^{*}$ line at $610.3 \mathrm{~nm}$ at a distance of $0.62 \mathrm{~mm}$ (dashed curve) and $10 \mathrm{~mm}$ (solid curve). The arrow indicates the region of self-absorption (small dark triangle). (b) Linewidth of the 610.3-nm line determined from the data in (a) as a function of target distance $z$. The shaded area very close to the surface $(0.5-0 \mathrm{~mm})$ indicates the region of self-absorption, and for $z>2 \mathrm{~mm}$ the values are limited by the spectral resolution of our system.

to more than $10 \mathrm{~mm}$, and two interesting processes can be observed that occur close to the target surface: line broadening and self-absorption. Line broadening can be caused by different mechanisms. ${ }^{8}$ Since Doppler broadening under these conditions is typically limited to the subangstrom range, Stark broadening due to collisions between $\mathrm{Li}^{*}$ and electrons dominates for plasma densities above $10^{14} \mathrm{~cm}^{-3}$.

We extracted from Fig. 3(a) the evolution of the linewidth with target distance $z$. The inset shows representative spectra obtained at two distances, each one obtained by averaging over two horizontal lines in Fig. 3(a). Strong line broadening can be observed very close to the target compared with a narrow line at a target distance of $10 \mathrm{~mm}$. The spectra were fitted to a Lorentzian function, whose width $\Delta \lambda$ was plotted in Fig. 3(b) as a function of $z$. For large distances the measured linewidth is limited by the resolution of the spectrograph to $\Delta \lambda \approx 0.5 \mathrm{~nm}$. However, for $z<2 \mathrm{~mm}$ a dramatic increase in linewidth can be easily resolved, yielding a maximum value of $\Delta \lambda=3.3 \mathrm{~nm}$ for $z=0.5 \mathrm{~mm}$. Because Stark broadening dominates under these conditions by electron impact, local electron density $N_{e}$ can be calculated directly from $\Delta \lambda$ with the relation $\Delta \lambda=0.2 W\left(N_{e} / 10^{16}\right) \mathrm{nm}^{{ }^{9}} W$ is an electron impact parameter, which for the $\mathrm{Li}^{*}(610.3 \mathrm{~nm})$ line is weakly dependent on temperature. ${ }^{8}$ Using this relation, we obtain $N_{e}=7.6 \times 10^{17} \mathrm{~cm}^{-3}$ at $z=0.5 \mathrm{~mm}$. This value can be compared to the one obtained in a recent study ${ }^{9}$ on $\mathrm{LiNbO}_{3}$ limited to a minimum target distance of $z=2 \mathrm{~mm}$. The authors employed a single-point scanning technique and reported a maximum value of $N_{e}=2.9 \times 10^{16} \mathrm{~cm}^{-3}$. Because $N_{e}$ is expected to decrease with $1 / z$, our value, which is more than 1 order of magnitude higher, is consistent with the much shorter target distance.

For shorter distances [shaded area in Fig. 3(b)] the linewidth cannot be measured precisely, because self-absorption begins to strongly affect the line shape, showing a dip in the central part of the spectrum. Self-absorption may occur when the density of a given species is high enough for light emitted by this species to be reabsorbed by the same species. ${ }^{10}$ Self-absorption of the 610.3-nm Li* line can be seen in Fig. 3(a) by the small dark triangle close to the target surface.

In conclusion, we have developed a novel technique that allows precise measurement of the spectral, spatial, and temporal emission characteristics of laser-induced plasmas by use of a single detection system. By applying it to the study of $\mathrm{LiNbO}_{3}$, we have demonstrated the system's sensitivity, its large spectral window, and its flexibility to switch between the 2D spatial and 1D spatial and spectral imaging mode. With this technique we have been able to separate populations of different species by their different expansion dynamics. It has also been possible to observe bremsstrahlung, strong self-absorption, and Stark broadening effects near the sample surface, allowing a quantitative determination of the electron density as a function of target distance, obtaining values of up to $7.6 \times 10^{17} \mathrm{~cm}^{-3}$ near the target surface.

G. Epurescu acknowledges funding by the European Union (HPRN-CT-2000-00160). We are grateful to Paul Tadrous for providing the software ${ }^{11}$ for logarithmic intensity representation of the images in Fig. 2. J. Siegel's e-mail address is j.siegel@io.cfmac.csic.es.

\section{References}

1. For a review see, for instance, the feature issue of Appl. Opt. 42, 5937-6225 (2003).

2. D. B. Geohegan, in Pulsed Laser Deposition of Thin Films, D. B. Chrisey and G. K. Hubler, eds. (Wiley, New York, 1994), Chap. 5.

3. D. B. Geohegan, Appl. Phys. Lett. 62, 1463 (1993).

4. V. Bulatov, L. Xu, and I. Schechter, Anal. Chem. 68, 2966 (1996).

5. E. Pérez-Tijerina, R. Machorro, and J. Bohigas, Rev. Sci. Instrum. 75, 455 (2004).

6. S. S. Harilal, C. V. Bindhu, M. S. Tillack, F. Najmabadi, and A. C. Gaeris, J. Appl. Phys. 93, 2380 (2003).

7. H. R. Griem, Principles of Plasma Spectroscopy (Cambridge U. Press, New York, 1997).

8. H. R. Griem, Spectral Line Broadening by Plasmas (Academic, New York, 1974).

9. F. J. Gordillo-Vazquez, A. Perea, J. A. Chaos, J. Gonzalo, and C. N. Afonso, Appl. Phys. Lett. 78, 7 (2001).

10. T. Sakka, T. Nakajima, and Y. H. Ogata, J. Appl. Phys. 92, 2296 (2002).

11. Bialith image processing software (www.bialith.com). 